\title{
Sleep in the Work of Marcos José Salgado, Author of the First Book of Physiology in the Americas, Published in 1727
}

\author{
Gonzalo Flores ${ }^{1 *}$, Carlos Manuel Fernández-Sánchez², Alejandro Valdes-Cruz ${ }^{3}$, \\ Julio Cesar Morales-Medina ${ }^{4}$ \\ ${ }^{1}$ Instituto de Fisiología, Universidad Autónoma de Puebla, Puebla, México \\ ${ }^{2}$ Avd. Portugal 56-3ㅇ A, Salamanca, España \\ ${ }^{3}$ Dirección de Investigaciones en Neurociencias, Instituto Nacional de Psiquiatría Ramón de la Fuente Muñiz, \\ México D. F., México \\ ${ }^{4}$ Centro de Investigación en Reproducción Animal, CINVESTAV-Universidad Autónoma de Tlaxcala, \\ Tlaxcala, México \\ Email: "gonzaloflores56@gmail.com, "gonzalo.flores@correo.buap.mx
}

Received 10 October 2015; accepted 24 December 2015; published 29 December 2015

Copyright @ 2015 by authors and Scientific Research Publishing Inc.

This work is licensed under the Creative Commons Attribution International License (CC BY).

http://creativecommons.org/licenses/by/4.0/

\section{c) (i) Open Access}

\begin{abstract}
Sleep and wakefulness are functions of the central nervous system (CNS) that are altered in numerous psychiatric disorders. Interestingly, Marcos José Salgado published in 1727 his book which is considered the first book of Physiology in the American continent and entitled "Cursus Medicus Mexicanus" (Figure 1) that suggests the use of drugs to regulate brain functions and their therapeutic action on sleep. In the work of Salgado, the concepts of sleep and wakefulness are offered using Hippocratic and Galenic knowledge to explain the pathophysiology of both functions. Today those concepts might be considered wrong. However, taking into account the historical moment and the intrinsic value of the text written in Latin, this manuscript is unique. Considered by art critics as valuable, there are only eight original copies of this book. In the present report, we discussed paragraph by paragraph the chapter "Sleep and wakefulness", the work of this Medical Doctor of the New Spain.
\end{abstract}

\section{Keywords}

History of Medicine, New Spain, Eighteenth Century, Sleep, Marcos José Salgado

\footnotetext{
${ }^{*}$ Corresponding author.
}

How to cite this paper: Flores, G., Fernández-Sánchez, C. M., Valdes-Cruz, A., \& Morales-Medina, J. C. (2015). Sleep in the Work of Marcos José Salgado, Author of the First Book of Physiology in the Americas, Published in 1727. Advances in Historical Studies, 4, 368-379. http://dx.doi.org/10.4236/ahs.2015.45026 


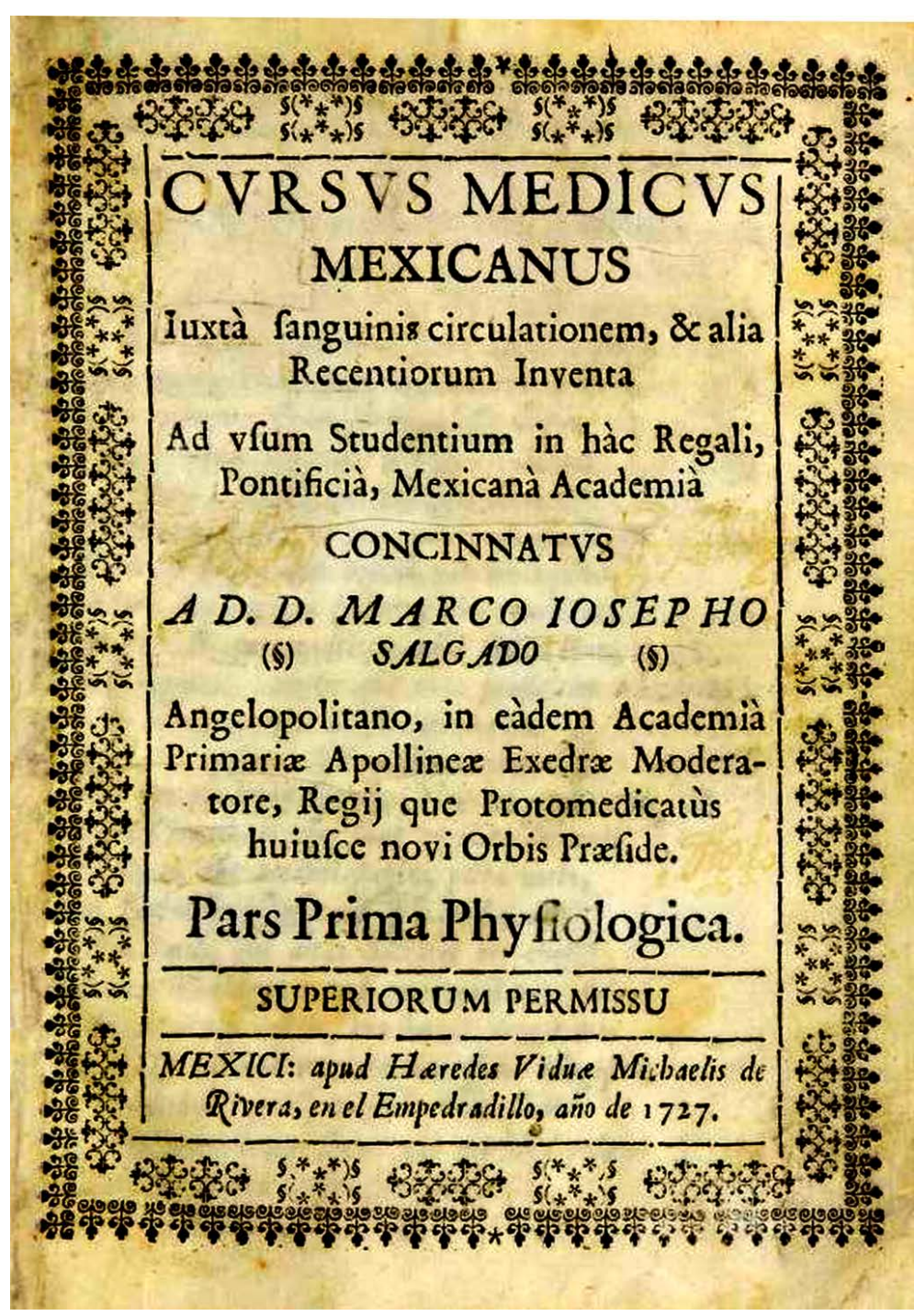

Figure 1. Cover of the book in the original language (Latin).

\section{Introduction}

Marcos José Salgado published the first book of physiology in the American continent in 1727. This text was written in Latin and entitled "Cursus Medicus Mexicanus" (Mexican medical course). This book communicates basic scientific knowledge, for example, describes the components of the living beings and the humors, to the complexity of the mind functions, including sleep and wakefulness. This last topic was discussed at the end of his work, with emphasis in the process of sleep (Salgado, 1727). The physiological concepts discussed in Salgado's book represent progress in teaching of physiology in the American continent as suggested by Izquierdo (1934) with certain historical delay. In this regard, the concepts on blood circulation by William Harvey (1628) were published and accepted in all Europe fifty years prior to the publication of this book, however, this knowledge was not broadly discussed. In addition, Izquierdo (1939) suggests that Salgado's work did not explain the mechanism of blood circulation present in Harvey's report (1628). However, as evidenced by De Micheli Serra (1983) and De Micheli Serra and Izaguirre-Avila (2010) and as we can see on Salgado's work, page 184, he cites the work of Harvey and clearly mentions that the mechanism of blood circulation had been described by Harvey (Salgado, 1728), but fails to explain the new concepts described in William Harvey's Book. Moreover, Salgado attempted to mix Galeno's and Harvey's knowledge related to blood circulation, even when those theories were impossible to merge. However, as mentioned by De Micheli Serra (1983) and De Micheli Serra y Iza- 
guirre-Avila R (2010), this issue has a rational explanation, Harvey’s book was not accepted by the Holy Inquisition until 1739. Therefore, if Salgado's report explains the complete blood circulation mechanism as reported by Harvey (1628), the Holy Inquisition may have sanctioned Salgado. For all these reasons, we believe that Salgado's work summarized and presented in one book the new concepts of blood circulation with the old physiological concepts of Hippocrates, Aristotle and Galeno. This book was used in the physiology course imparted to medical students of the Royal and Pontifical University of New Spain, now known as the National Autonomous University of Mexico. Moreover, this book has recently been classified as rare (Finch, 2005), as there are currently only eight original copies written in Latin, six in the Americas and two in Europe (Finch, 2005). There is not a translation to another language from the original Latin, such as Spanish or English.

\section{Sleep and Wakefulness}

The chapter related to sleep (treated II, section four, de somno, et vigilia, p 296 to 310) is divided into several sections or paragraphs. This chapter describes the concept of sleep, and its opposite wakefulness. In this chapter, Salgado also relates functions with the senses: vision, olfaction, taste, touch, etc., and literally says:

"What is the essence of sleep? Is it the closest and ordinary unwillingness to use the external senses? Certainly, it is said about of the ordinary, as if from a separate order of nature, so that extranatural common types of sleep, such as apoplexy, lethargy and similar pathological conditions. Indeed, such unwillingness or natural blockage for feeling is explained extensively in the texts of classical authors. Aristotle called sleep, the first link with the senses, or the union or decrease of the natural indoor heat; Galen, however, in numerous texts, relates sleep with the causes of the "symptoms" (last chapter and afor. coment 3) and he has called brain cooling o rest of the animal faculties. Then clearly, the authors are divided between several views. Who consider that this entity is positive and sleep is a deprivation function; whose opinions are a probability not accepted until present, we consider that sleep is that state of the living body in which all the senses are drowsy and tied; while wakefulness, in opposition, is when the senses are loose and free to sense".

Subsequently, he raises numerous physiological concepts about sleep and wakefulness mechanisms valid at the historical moment. In addition, Salgado tries to make an association between sleep and wakefulness, the participation of the senses, and the role of the spirits taking, in account the Galenic school. He suggests that the food arrive to the liver, there it is transformed into natural spirits. While they became vital spirits in the heart, these are transformed in animal spirits in the brain. Izquierdo (3, page 78) suggests that Salgado only considered animal and vital spirits. Interestingly, when Salgado wrote about the "steam of the food", he never mentioned that this is a natural spirit. In addition, he mentions the importance of tension and relaxation of the nerves of the brain with sleep and wakefulness, now known as the neural transmission. This is reflected in the following paragraph called "The real cause of sleep".

"However, what process produces the union between sleep and wakefulness, at this moment is unknown. Some authors suggest that the brain ventricles are sometimes obstructed by vapors of food and the influence of the spirits to the organs of sense are blocked, and hence the vapors dissipated and resolved. When sleep is over, wakefulness is back with common (animal) spirits. Others authors, argue that sleep is produced to maintain relaxation of fibers of the brain, which by the tension, are eligible to receive and perceive impressions coming from the sense organs to the seat of the soul. So that if the terminals of fibers (ropes or cables) are loose or no tight, they will not receive the impression of movement. However, if they are tight, they let a fast propagation. Interestingly, the mechanisms by which the fibers of the brain is relaxed and lack the natural tension, is due a provision of the animal spirits. For if the fibers of the brain, and the tubules constituted thereof, fail even a little, or their special nature be altered, or they be disabled as by a mixture of vapors, or torn asunder, they will not extend as much as is needed".

With Galenic arguments, Salgado raises the contributions of animal spirits in mental functions and includes the participation of various environmental factors such as labor, type of food, etc., in the physiology of sleep and wakefulness. However, he manages these two terms as extremes. Interestingly, he attempts to explain the use of narcotics or drugs to induce sleep. In the next paragraph, entitled "Verification", the sleep concept is explained:

"Based on the above, excessive work or an abundant meal produces a large amount of spirits which easily induce sleep. It is natural that spirits absorbed or generated by overwork or an excessive meal are made fatter by the vapors of food and drink, especially at first; however, if someone works or is full of food and drink, and immediately tries to sleep, they may not be able to sleep easily at first because of the abundance of spirits. When 
these spirits are abundant, the body feels vigorous and ready, and they do not diminish quickly with the vapors of food and drink, but have some force, which is not sufficient for the presence of wakefulness. Unless great vehemence be the cause that may be able to weaken the power of wakefulness, such as seen if one takes narcotics, which bind to vehement spirits with particular force and inspire inactivity in themselves such that no applied care may be able to rouse one from deep slumber, and they may sleep forever".

Today, sleep and wakefulness remains a complex function and, after nearly 300 years, we have not been able to fully understand this process as Salgado had not. With major advances in electrophysiology, neurochemistry and molecular biology, each day we are closer to better understand how this process occurs, however, there are still gaps in the knowledge of this field to be elucidated. Moreover, Salgado involves sleep and wakefulness within functions of the soul suggesting that both functions are interconnected with the soul and thus gives an active role to the process of sleep.

Here we present what he mentioned in the section called "Some views on the nature of sleep".

"While we have entered into this most difficult field (the nature of sleep is certainly very difficult to understand completely) it will not be out of the question which are clearer, and even self-evident, such that they cannot be denied, let anyone address it, because after it is more plausibly understood, the nature of sleep may be confirmed In principle, it is true what Aristotle had taught in his book entitled "Sleep and Waking", especially the action to block the senso-perceptive faculty; because as wakefulness is the exercise of the external senses, and also, of voluntary movement; Sleep, therefore, is the deprivation of the external senses; and those things that are considered as positive or negative necessarily rotate about the same object or subject; and when sleep and wakefulness are considered as disease and health; and as well as vision and blindness, hence it is evident that we must consider sleep and wakefulness as perceptive abilities as well as free actions; and since the act of perception may neither be considered only respecting the soul, nor only the body, sleep and wakefulness should be considered phenomena namely that not only the soul, but also the body, works through wakefulness, and similarly not only the body, but also the soul for the most part, rests through sleep".

Later on, Salgado again emphasizes the strong connection between sleep and wakefulness, he sees them as opposite functions. A critical point in both is that he says that the spirits are transported by nerves and highlights its role in waking and its absence in dreams, as we suggest on nerve transmission at present time. These ideas are described in the paragraph entitled "What is required for the wakefulness".

"However, to understand sleep and wakefulness must be established a connection with each other; because if you understand what sleep is, in itself consists, in opposition to what is wakefulness, and likewise understand the nature of the vigil, accordingly the knowledge of sleep arrives easily; therefore, as nature manifests more wakefulness than sleep, your knowledge will investigate the nature of sleep".

"For all it is clear that when a man is awake, he is willing to perceive sensible objects, and distinguish them, and discourse about them and regulate his motor behavior that depends on the discretion of the will; and when that fails, it is certain that the man will sleep instead; when operations corresponding to wakefulness, are not well made, nor abundant, and the animal spirits act strongly on the brain, and they cause a violent strain on it, covering it and passing through those nerves, the passions are marked external to the spirits and communicated by them to the immediate spirits, and transported to the seat of the Soul, which is the organ of sense, first determined or qualified form that received with certain modifications, objects to the soul itself make out the sense, perceive the objects, and distinguish the differences; this is unchanged, since waking requires abundance of spirits and paths, that is, the arrangement of nerves, by which the same spirits are transported and maintain the proper tone, and hence it follows that during sleep, mean spirits coincide with the laxity and collapse of the nerves which impedes the influx of regular spirits to the sense organs".

Salgado suggests that wakefulness is related to, but not dependent on the sense organs. In addition, he talks about its relationship with common sense, which will be animal, cortical activity. In the paragraph entitled "Sleep and wakefulness are passions of the brain", Salgado clarifies that the brain and no other organs are directly involved in these functions.

"As already mentioned, wakefulness is an innate ability to exercise the external, and common senses; instead, they are disabled or attached by certain natural necessity with sleep; thus, if some perception were available, by which an animal would perceive a particular object, then it is agreed that it would be awake, and similarly if all external senses were bound which ordinarily were free, and would inevitably sense the motion of external perceptions, and distinguish objects among them, these would be called wakefulness and sleep, which cannot take place simultaneously. Consequently, it must be established that, in wakefulness, both external and common 
senses are presented. In opposition, in sleep, external and common senses are not working together or both are tied to the sleep state. As mentioned before, common and external senses, depends on brain disposition. Because common sense lies in brain function and strength of the external senses drift of the common sense. Consequently, both wakefulness and sleep, are passions of the brain and not of the heart, as mentioned by Aristoteles".

It is clear in Salgado's work that sleep is not merely the absence of what he calls the transport of spirits and is now known as nerve transmission. Indeed, during sleep a series of processes occur in the brain. This process that links the perception of the senses passions [now known as rapid eye movement (REM)], is the period in which the short term memory is consolidated to long term memory. Salgado also speaks of sleepwalking, without addressing his understanding. This paragraph is what he calls "In the dream works imagination".

"It should be noted that sleep is tied with the common sense. As sleep perceives the passions of the external senses, not in terms of its own passions, which are, independent of the external senses. Because the passions are produced into the sleep. In addition, the movements present in sleep, as in sleepwalking. For example, in sleepwalking, while a person sleeps and walks, the person does not perceive the passions of the external senses, or judge over them, but he certainly sleeps. However, the imagination is working in sleepwalkers, they are represented in a fantasy of what to do, to whose attainment strongly tends to the man, looking and comprehensive means for performing. The ability to walk does not need the work of the external senses that he cannot perceive due to the lack of spirits and lack of tension in the nerves. In addition, this state fully understands the alleged confusion, between common sense and fantasy, as if considered distinct qualities. It is easier to say that common sense is tied completely, whose strength is to perceive the passions of the external senses, keeping free from fantasy, that include the relation with ghosts, which represents and perceives, even with the external senses off'.

The paragraph called "the cause of sleep and wakefulness is explained". Here, he proposes the characteristics of somnolence and tries to explain the physiological changes that occur in somnolent people as well as the role of wakefulness, and its opposite, sleep. It is interesting that he speaks of pores in the nerves and how that energy is transferred through the nerves not only into the brain but throughout the body. In addition, he distinguishes so what is now known as the central and peripheral nervous system.

"Similarly, the causes of sleep and wakefulness are recognized by another direct path; the cause of wakefulness is, as he said recently, the abundance of animal spirits in the brain that influences the nerves, which fill, put in tension and make turgid. In opposition, the cause of sleep must be the lack of spirits in the brain and nerves, with unswelling and laxity of these that we observe in the sleepy with the knee falter, the wobbles head, and eyelids almost closed. Consequently, the whole body is flabby; and this confirms the sleep state, commonly accepted by all: that is, the repair of animal spirits, which are lost during wakefulness, so again, the person own operations begin. It is not difficult to understand, the way in which the shortage of spirits produces sleep. Considering that the brain, in wakefulness, is full of nervous juice, and spirits, and porosities are filled with some expansion, and fullness. Therefore, the transfer of energy easily passes with impulse to every nerve, not only to the external senses, but also to all the body's nerves. Since the spirits are not interrupted, but are together to form one body. In this state, the spirits are abundant in the brain, they are going to push and they will be in contact among them. So, when a shortage or lack of spirits occurs, in consequence the opposite occur: the brain becomes certainly flabby, nerves derivatives of the brain are relaxed and subside; to some extent, become clogged, and the sides of the nerves touch each other. Therefore, the loss of spirits into the brain cause a lack of influence and indisposition of the organs to perceive external objects, hence, sleep occurs".

In the next paragraph entitled "Food-induced sleep-for its vapors" describes that not all the foods have the same effect to induce sleep. In addition, he emphasizes that milk and wine are better to induce sleep than other foods and points out the effect of alcohol as a sleep aid. He highlights how the sleep process could be carried out. Salgado's work continues the misconception that food vapors cause the entire process. A successful point is when he mentions that nutrients from food reach the brain through the bloodstream and that the role of sleep is important for the regeneration of the brain. However the knowledge about the physiology of sleep and its opposition to wakefulness is presented around the same basis: spirits. The text deals with:

"Not only continuous sleep is induced with a decrease of spirits, but also with abundant food intake, especially vaporous nutrients, as well as a beverage intake of the same nature, mainly milk, wine, and liquor, all with abundant spirits; because all sleep-related factors must be connected. The lighter parts of the food and drink are easily digested and integrated to the mass of the blood. Once in the blood, these are carried by the circulation to reach the head. In the head, they sleep into the cortical substance of the brain, after they penetrate 
to the spinal with great abundance and stuff their porosities; when the porosity is completely filled, closure is prevented, as well as the movement of spirits and the influx of themselves; until this portion of food and drink is dissipated by sleep or the aforementioned internal heat, and the power of the spirits approaches that of newly modified spirits in the cleaner part of the blood, and their amount restored with new impetus as is the nature of spirits; which opens obstructions and access to the organs of the senses, so that a man begins to be awake. Other times, how the substance of the food or beverage are transported to the cerebral cortex, or spine substance; to this extent, only vapors are carried to the brain by ways unknown or even by the substance of the nerves itself; but when they are obstructed, then, sleep begins; unless it appears a compelling reason, the vapor of the food and drink is mixed with the spirits, continuously up and down, and somehow dissolved in the corporeal nature, released by wetting and cooling; with whose laxity, perceived in spirits, they become flabby and weak; and their nerves, perhaps their pores, reject the tension and appeases; understood, it is not difficult to see how sleep with obstruction caused by food and drink is produced. With this reasoning, Galen said, "sleep is the over-regeneration of brain".

The following paragraph is rather repetitive in its content, entitled "Why narcotics produce sleep-the sleep by weakness." It begins by mentioning the different types of temperament (explained at the beginning of his work and out of the scope of this review). He makes a relation of the temperaments with sleep and wakefulness. Also he mentions the effect of heat and cold on sleep and finally tries to explain the effects of narcotics, stopping the mobility of what he called animal spirits.

"With these assumptions, it is easy to understand, the way narcotic drugs work, cold things carry excess and induce sleep; these medications cause the arrest of spirits and as soldiers in the brain, they are removed, decreased or, at least, their influence on sense organs reduced. These actions can be modulated in two ways, either with a tiny amount of spirits, or by low temperature thereof, which prevents its movement. The amount of spirits decreases with poor meals, overwork, or a natural or extra-natural surplus of frigidity; for that reason sleep is incurred in the hungry and those who are very famished; likewise the elderly are as somnolent as they are pituitous on account of a defect of the blood and frigid temperature; Certainly, the spirits are altered with cold food or drink, as it was mentioned before about the humidity; as cold prevented the movement of spirits, therefore if they are hotter, they are more mobile, if they deviate from heat are less agile for movement; for these three reasons narcotics, or they may be called drugs, or poisons, induce sleep; when they are used, they act with opposite actions on the spirits, disordering these with excessive frigidity and a mysterious force and stop and prevent the movement of spirits".

In the paragraph entitled "Benefits and harms of sleep and wakefulness", Salgado talks about sleep and wakefulness and how these processes must be in balance and its absence or exaggerated presence may be harmful or cause illness. He suggests that both protect life; and also highlights the protective role of both for the body in general.

"Having explained these arguments superficially, as they are discussed with more specific details in another place. The task is to inspect what benefits or detriments either sleep or wakefulness to the human body. In principle, it is true that sleep and wakefulness every occasion presented, help to protect life, which are not only useful, but necessary; however, if these passions were uncontrolled or unwelcome, they may harm the body, as Hippocrates, Aphorism 2 text 3 said Sleep and wakefulness, either in excess is bad" and when the text 4 or satiety nor hunger, nor anything else that is out of the natural, is good; so both sleep and wakefulness, should have the average, and thus maximized; as a moderate sleep restores, humidifies the organs of the abdomen, gets fatigued members, renews the spirit, helps digestion, restores fluids, rest, softens the acids moods, thick soft, and stops excessive movements. Similarly, moderate wakefulness excites heat, reduces moods, directing the waste to the appropriate channels, and promotes optimal distribution of food".

The next paragraph that, despite the title "The sleep time-The force of habit in sleep, Impairment of meridian sleep” does not add much specific information. Although Salgado mentions the importance of sleep in the night time, he does not mention the existence of a circadian rhythm. Moreover, he talks about the consequences of prolonged wakefulness, but he fails to make a connection with psychiatric disorders. Finally, he mentions the states of sleep during the day, but only related to headaches, without correlation with other types of disorders.

"In opposition, when sleep is longer than it should, it causes apathy of body and soul, reduces the natural movements of the bowels, fills the head with vapors, retards the movement of blood and all liquids, and reduces all the senses, especially internal, and produces all those bad things mentioned recently on leisure. Similarly, uncontrolled wakefulness exhausts the spirit and blood, dissipates the forces and induces weakness throughout 
the body, which may originate more serious ailments such as indigestion, fever, lack of mood, weakness forces and others similar things".

"Clearly, the appropriate time to sleep is at night according to the usual custom of men; somehow silence, gently encourages night sleep. In addition, the absence of the sun, minimally distracting the senses by the little natural lighting, induces a sleepy state easily. For these reasons it seems that night time is the time suitable by nature to rest. Therefore, the objective of sleep at night is to reduce fatigue and exhaustion due to vigilance and work. In opposition, long time without sleep or sleep at a different time that at night, no doubt, with time is harmful. An appropriate document is the Hippocrates 2, prognosis, text II, that indicates when a person spend sleepless nights due to night work, or engaged in other works, and sleep in the morning or at noon, the health state is apparently without loss. However, by custom, sleep during day is regarded as unnatural. In addition, it should be avoided afternoon sleep, special in those people exposed to ailments of head, or noon sleep when the head is full of steam, the brain is affected, especially if sleep was unaccustomed. As taught correctly Avicenna and the Scola Salertiana:

Have a short or no sleep meridian:

Fever, colds, sloths, headache:

This will induce the meridian sleep

The afternoon sleep makes heavy head and it is inadvisable, except only in cases, after the tiredness and fatigue from work or by prior wakefulness; since this type of sleep are helpful, increases relaxation and replaces worn forces".

In the next paragraph called "A doubt-different measures of sleep" Salgado does not provide relevant information. He only indicates the number of hours to sleep. At the end he suggests that it is necessary to sleep six to seven hours a day, but he does not give a scientific explanation for this. However, it relates more to the manners, that the brain physiology at rest.

"They have asked many times, how many hours should healthy sleep be extended? About this, many argue that an adequate time is six or seven hours; and in truth, it is an average measure, sleep cannot be too long or short; in the same way it is not possible to suggest a unique amount of hours to sleep, for every person, therefore it is not possible to apply the same rule to all of them, some people need a longer sleep, other people shorter, as the different habits of body, food, and drink, use and type of exercise. Similarly, age and seasons of the year, also have an influence in the hours of sleep needed; consequently, for children perhaps a more prolonged sleep is suggested, while the young needs perhaps a shorter sleep; in winters is suitable a more generous time, but in summer, it is necessary a shorter sleep, as mentioned in the aphorism 15. libr. 1:In winter the intestines, indeed by nature very hot, the sleep are longer, who work hard during the day, need quiet night, in opposition, people with a few hours of work need a shorter night, and finally, for health, people with obesity should sleep more hours compared to thin people".

In the next paragraph entitled "The supine position is harmful.-For whom the prone position?" he proposes different sleeping positions and discusses them relating them to the anatomy of the abdominal organs and digestion of food. The paragraph also mentions the relationship of pain, heart or liver problems with sleeping positions. Finally, he erroneously notes that supine sleep is harmful because it does not allow proper function of various organs of the chest and abdomen, without highlighting the possible pathological processes that might occur.

"The question also raised is, what will be the healthiest way to sleep?; that is, if the body should be face up (supine position), on either side, and between them whether on the right or on the left. - Avicenna in the lib. First, sen. 3 doct. 2, chap. 9, shows that the onset of sleep should be on the right side; in that position the stomach is better placed by the liver and also helps to the chylopoiesis; in addition, this position helps the processing of food; however, the last part of sleep must be carried out on the left side; it assumes that the digestion is over, and now the work is that the chyle slip through the pylorus to reach the intestines; since the pylorus is on the right side, the position on the right side prevents the output of chyle from the stomach cavity, especially from the bottom; and although denominated always improbable by Avicenna, especially in a healthy man, that present well constituted intestines, without infarction or no obstruction, must choose either side initiating sleep, as taught everyday experience, acquired by reason of custom and especially the comfort or discomfort perceived as posture, which occurs with discomfort or illness, although sometimes is not evident, must be replaced; because a healthy man sleeps good in any position".

"People with diseases, however, have a posture (position), there is not a particular posture for a disease, or 
pain that prevents any posture, in one or another position to prepare to initiate sleep; as manifested in hypochondriacs or in those with heart pounding, who find the left side posture difficult and upset; in contrast, those with enlarged liver or with an inflammation of the liver, they do not support to sleep on the right side, and the same occur with a wide variety of conditions to sleep; for healthy people, it is difficult to impose rules to change the position during the progress of sleep, because sometimes we are not in control with the sleep time, many people end the night with a single sleep, in the same position; however, who often occurs, that excited during sleep, it is to consider when sleep occurs on either side, because that would be better both the development and distribution of chyle, and with less fatigue to the body. In general, people should avoid the supine posture (face up), which impedes breathing and the correct work of the kidneys, also this pose does not help the stomach for digestion, heart oppression occurs and is lying; it is possible that these latter effects are more applicable to the face down position (prone position), because of the oppression of the heart and diaphragm, because the spine is pressing on the chest; however, such a prone position, rarely required by nature itself, may allow patients to find some relief, when there is weakness in the stomach and abdomen, and pain in the intestines".

The paragraph called "Sleep should be avoided in a state of stupor-To whom should it be prohibited?" This paragraph regards the need to use sleep-promoting drugs or some procedures in the treatment of some disorders. Knowledge about febrile processes, as already mentioned above, highlights the need to avoid sleep in stages of sweating, and the need to sleep during the flu to recover the health; however, he asks the question because sweating occurs in febrile processes.

"The same natural evaluation, and the opposition law, hints to who should sleep or be awaken in diseases; because there should be no doubt that in patients with excessive sleepiness, lethargy, stupor, coma, and stroke, sleep is harmful and allow this, it results completely harmful and disastrous; when the disease is more intense, must completely focus to promote wakefulness using various techniques, even painful drugs; in contrast, in case of delirium and over-wakefulness, sleep must be reconciled at any cost, even with hypnotic and narcotic pharmaceuticals; sleep should be prohibited in case of internal inflammations to prevent access of external things to inward; similarly, those who suffer from sweating, such as typhoid fever and elodes fever, it is not adequate sleep; because it is clear and has experienced, that the sweat flows more abundant during sleep; in the beginning of the accesses to fever, mainly with the chills, sleep should be completely avoided, because of the danger to suffocate, or because some time is prolonged fever attack; however, sleep should be allowed, even seek, as indigestion, flows, colds, especially when they are accompanied with pain and trembling, and when sweat is desired because it helps to restore forces".

In the next paragraph, which he calls "A doubt, in the worsening, interferes sleep. Equality for food” continues his description of febrile processes. He explains features and gives examples. Also he explains why sleep should be avoided in processes as fever. As mentioned before, we should favor sleep in lapses without fever to recover health. Also, he raises the association between fever, sleep and food with their features.

"As often occurs, intermittent fevers may come to be so long such that no perceptible interruption may be noted; on the contrary, before one onset runs out, a new one may arrive; such fevers are rightfully called "subintrantes," those that enter stealthily; during this, the sick may not be allowed or able to attain sleep without fever; you must understand, since the fever lessens in time, one may be able to sleep free from nuisance. Indeed, it is considered that at any time fever is harmful; at first, as I said, the dangerous fever may cause suffocation; when the fever increases, it can make the problem worse, due to the reduction in breathing, which is also reduced during sleep; and the rest state also reduced the breathing. Neither in this state, because then it is time for evacuation, and all sleep contains evacuations beyond sweat, the pursuit of which causes a decline, nor when the decline remains free as befitting administered sleep, can the fever be eradicated; while anything else may be especially inevitable, lest the forces certainly collapse; it is necessary to establish that if sleep lasts a short time, it should be considered harmful; it is certain that a rise in temperature (fever) should be passed without sleep; however, when an interval is observed, with no fever, it will be time to sleep, and a similar rule was established to eat, that is, in the far time from the start; so that care must be taken over this period than any other, and if there are enough time to eat and digest the food; if the interval was short, or if there is no interval between two periods of fever, in the final state; it should be consumed soft food without fats. In addition, sleep should be brief, due to, both, sleep and food with fever manifests with weakness and also affecting natural forces. They are harmful, however, they should be avoided as far as possible, when in doubt, consult what have been written the brightest medical practitioners".

The paragraph labeled "Prognosis from Sleep.-The optimal posture and faulty posture" is a continuation of 
postures during sleep, as the paragraph mentioned before. In this paragraph, Salgado speculates that posture may indicate pathologies such as those related to delusions. Its primary approach of a relationship between certain positions and certain disorders, such as torticollis (wry neck), causing asimmetry in head posture, but in other cases there is a lot of speculation.

"Sleep has not only a reason of cause, but also it has a signal; what different meanings are assumed, based on their qualities, and only suitable for prognosis; what myth does Hippocrates teach?, and called them, which qualities are assumed from the duration, quiet or disturbance of the sleep, here we only examine the signals that are responsible for the position on the bed of sick people; taking into account what Hippocrates first analyzed about the sleeping position of sick people, and as he suggests, we must suppose, what position should be convenient and healthy in such diseases?, what position should be used in a healthy state, in which it deems best, one in which a person should sleep on the right or left side, head, hands and feet slightly bent or placed in a free position; are bad, as already said, rolling onto your back (supine), hands and legs spread apart and; even worse if the person is inclined, feet hanging off the bed ("rises running"), and if, by chance people are naked and without clothing and warm or frigid, it is considered a bad sign and still worse if they are cyanotic with their hands also in the same way, be declared the disease, without delay? or with serious delay?, when occurs an asymmetry of the head (neck) by a deformed position; since those positions to be in the bed indicate an internal turmoil of the intestines, and foreshadowing of inflammation, or sometimes delirium, or mean the dismal state of the blood due to an insignificant solution or clotting same and maximal consequent loss of strength".

Interestingly, the information used in the paragraph below entitled "Question._-Warning._Correct decision.When to sleep?, Exceptions” since it raises first, some pathological conditions may be exacerbated with abnormal sleep positions, for example, respiratory disorders. In addition, he suggests that some signs during the stage of sleep may be associated with some brain disorders such as seizures. On the other hand, he makes a direct link between movement, evacuations and the use of laxatives. It is clear that the person must be awake with free movement to obtain better effect of the laxative. Finally, he proposes the effects of some purgative or laxative pharmaceuticals on sleep, their presentation types and their characteristics.

"The fact that sick people sleep with the mouth open and yawning, this position indicates difficulty in breathing and a lesion in the organs related; in addition if a person sleeps with the eyes not completely closed, or if the lips are in constant movement, we should suspect a disease related to the brain, and irritated nerves, from those symptoms, we should expect an imminent convulsion; all these symptoms are signs of brain damage, these behaviors are far from the ordinary and natural state; similarly, if a sick person want to be sat often, it is a bad sign, this sign is specially bad in thoracic disorders, in these disorders a difficulty in breathing, and the orthopnoea often seen in the parapneumonic is clear; then fast movements of the legs, the possibility to leave the bed, undress themselves, are clear and strong evidence of internal inflammation".

"The last deserving knowledge about sleep is to know when is convenient to take a sleep-inducing drug. This topic is discussed frequently and practitioners have observed carefully that after a person intake of a drug, the person should be monitored constantly, the reason is that sleep stops or delays body evacuations as indicated in Hipp. 4, aform. Tex. 14 and 15; "after the intake of Hellebores to induce evacuations, the body has to be in constant movement; in contrast, in order to delay evacuations, the body has to rest; however this common practice is not universal, the practitioner must distinguish the times of action and the form of the medication; the drug could be liquid or solid and thus affect the delivery of the drug, with these common warnings, the accepted practice indicates that a liquid cathartic can prevent sleep, whereas after a solid cathartic, especially a pill, sleep is recommended until evacuation begins; following this, one should be awake, lest the movement, having begun, be halted; indeed, this method is rational and fortunately corresponds in practice. The practitioner must then monitor the patient, one the first evacuation occurs, the person should not go back to sleep; the rule (should be ) that after the intake of a liquid drug a person should be awaken and after a solid form is taken a person can sleep is not universal; for example, if the liquid drug were strong and energetic, sleep is not an impediment since a mild sleep does not reduce the effects of a strong medication, it even helps it to work better; similarly, if pills had a weak effect, they may prevent sleep; lastly if the sick person that will receive the cathartic has a weak stomach, this person can reconcile sleep, the sleep should be shorter in this person after a liquid drug and longer after a strong drug”.

We have to exempt to this rule constipated and people with abundant fatty juices in the first region, these people have to be not only awaken but in constant movement, as previously discussed by Hippocrates in the aforementioned aphorism where he shows the same practice in the use of cathartic drugs called potions that induce the movement of the body; the actions of these drugs are in agreement with the present manuscript, after a 
cathartic drug start working in the body (and after its actions are terminated) the sick people must remain at home and at rest; the practitioner must monitor whether the patient is dizzy or with vomit; in this case, rest and sleep are important to improve the recovery; in some cases, it is convenient to use an "anti-method" where a cathartic drug is dissolved in food or inviting beverages to hide the nature of the pharmaceutical.

In the last paragraph "Another reason to induce vomiting.- Narcotics should be administered cautiously in breathing diseases," He explains the characteristics of drugs to induce vomiting and its physiological effects in producing it. Finally, the text gave several preventive measures in terms of sleep-disordered breathing.

"I should have a clear mention about drugs with descaling effect in the stomach and intestine. The drug should be dissolved, transported and mixed with the juices of the body; because in the case of vomiting, the content is different compared to the food, as they have a more irritant and disgusting effect, given by the stomach itself, and its membranes. This effect is observed immediately. In some cases, these drugs disturb sleep. In weak individuals, their strength is lost, while strong individuals, during sleep time, uncomfortable and inconvenient symptoms appear, as presented pickets, heartburn type pain. So during sleep, drugs that induce vomiting must be avoided. For other fluids, such as urine and sweat, sleep is advantageous and should never be avoided. In opposition, wakefulness is extremely necessary for sputum excretion, because in sleep, sputum secretions accumulate in the respiratory tract. Therefore, expectoration (spitting) is required to prevent respiratory diseases. Consequently the soporific drugs and opioids should be avoided. The same should be done in whooping cough. Everything said about sleep and wakefulness is enough".

\section{Discussion}

The physiological concepts raised by Dr. Salgado and taught to his medical students were kept in his book (Cursus Medicus Mexicanus). After the death of Dr. José Díaz Brizuela, professor of physiology at the Royal and Pontifical University of Mexico, the position as professor of physiology was available at 1722 . As mentioned by Dr. José Joaquín Izquierdo (1934), Marcos Jose Salgado was the first professor of physiology (Prima of Medicine) who won this position as professor by real opposition. Prior Dr. Salgado position as professor of physiology, the Medical Doctor of the acting Viceroy obtained this position by pretended opposition. Surprisingly, Dr. Salgado got this position without been the Medical Doctor of the Viceroy. He was then a professor of physiology at the Royal and Pontifical University of Mexico between 1722 and 1740 (Izquierdo, 1965; De Michali, 2004). His book is divided into two volumes, the first consisting in six chapters and the second in six sections. His book is considered as a pioneer of Mexican physiological research. It was until early last century when pioneer research was done in physiology and related fields in Mexico. Indeed, several historians have considered Dr. José Joaquín Izquierdo, Dr. Arturo Rosenblueth and Dr. Fernando Ocaranza (Izquierdo, 1934; De Micheli Serra 1983; De Micheli Serra \& Izaquirre Avila, 2010) as the renovators of this discipline. In addition, Dr. Fernando Ocaranza for his political role and as promoter of reforms to teaching programs to include laboratory demonstrations at the time he was Director of the School of Medicine and Principal of the National University of Mexico. His influence allowed the development and growth of the Department of Physiology at the Old School of Medicine of the National University of Mexico (Izquierdo, 1934; De Micheli Serra 1983; De Micheli Serra \& Izaquirre Avila, 2010). While Dr. Ocaranza played a critical role in both, Dr. Izquierdo and Dr. Rosenblueth did a research stay outside Mexico in the prestigious laboratory of Dr. Canon at Harvard University (Aréchiga, 1988).

To fully understand what Dr. Salgado wrote on the topic of sleep and wakefulness in 1727, we should read his complete text. However, if we understand the concept he had about of what is a spirit, it is possible to approach the concepts that were handled before the William Harvey's book on blood circulation was published. The Harvey's book replaced totally the old Galenic concepts. Unfortunately, it took nearly a century for the work of Harvey (1628) to be accepted or recognized even in Europe. There were several causes: first it was written in English, not the dominant language in science at the time, and second, several researchers at the University of Padua claimed that these concepts were partially taken from the work of Jeronimo Fabricius, professor of William Harvey (Izquierdo, 1965). Third, the text had to wait to be translated to other languages, such as Spanish, Italian, etc., so that this work was known throughout Europe and the Americas. Therefore, we believe that the work of Salgado was a transitional material within the medical sciences as it tried to combine or raise what Galenic and Hippocratic school said about the functions of the human body and revolutionary advances on blood circulation. Apparently, Dr. Salgado read Harvey's work but not used as a base in his work, since such concepts replaced the ancient medical concepts; so he failed to understand the significance of the scientific revolution in- 
duced by the work of William Harvey. Finally, it was not until 1738, when the Holy Inquisition in Mexico allowed the work of William Harvey to be accessible to medical doctors through the National Library.

In sleep and wakefulness, Dr. Salgado addressed the topic in an orderly fashion from concept to its relationship with certain pathologies and the use of drugs at that historical time. He showed pre-Renaissance and Renaissance concepts, with concepts given in William Harvey's book. Salgado makes a clear point: the need for more research on the subject, not only at the level of a sign, which is present or absent in a disease, but also as a therapeutic tool in certain disorders such as febrile state. He also talks about the relationship between sleep and mood, considering the soul and the human will and not only changes in the activity of the sense organs. Dr Salgado addresses the issue comprehensively, but he makes several errors, mistakes from lack of access to information developing in Europe. Accordingly, the statements by Dr. Salgado are appropriate to the time, and the book makes his clinical experience clear, as their descriptions of the illnesses associated with sleep are very punctual. It is noteworthy his comprehensive view of the phenomenon of sleep as part of an ongoing process that must not be separated of wakefulness. It is noted as the theories of sleep indicated that sleep was a passive activity that depended only on the absence of wakefulness until early last century. Hess in 1944 (Hess, 1944) managed to induce sleep with electrical stimulation of the thalamus and posterior hypothalamus. Therefore, he established the foundations of the current theory of sleep, which considered sleep as a particular physiological state, actively induced and controlled by specific structures.

Interestingly, the theory of Dr. Salgado on the movement of spirits and sleep, i.e. of moods, is close to the idea that raised by Pieron in 1913, who proposed that during wakefulness a substance called hypnotoxin accumulates that induces sleep. While Pieron's works were highly discussed, it was later shown the existence of peptides that induced sleep. Another point made in Salgado's work is the effect of an increase in temperature during sleep. In agreement, Jouvet demonstrated in 1988 (Jouvet, 1988) that hypothalamic and bulbar systems were responsible for regulating the temperature and participate in critical mechanism of installing REM sleep.

Another point to note, Dr. Salgado discusses what the best position to sleep is, with particular emphasis that it does not interfere with breathing. This does not mean that Dr. Salgado is a pioneer in the study of sleep apnea but breathing, from its comprehensive vision, it is essential for a good sleep.

Finally, assertions about the process that occurs during wakefulness as food, rest, work, etc., and how they affect sleep. The fact that Salgado's findings present coincidences with later reports gives more value to Salgado's work considering the time and environment. So that Salgado's work presents several scientific concerns, which would be addressed later with the advent of novel techniques and knowledge about of the brain in the last century.

\section{Acknowledgements}

We thank Travis Miller for proofreading this manuscript. GFA and JCMM acknowledge CONACyT-Mexico for membership.

\section{Sources of Funding}

Funding for this study was provided by grants CONACYT grant (No. 129303) and PROMEP (CA-BUAP-120) to G. Flores. None of the funding institution had any further role in the study design, the collection of data, analyses and interpretation of data, writing of the report or in the decision to submit the paper for publication.

\section{Conflict of Interest}

No conflicts of interest on the part of the authors.

\section{References}

Aréchiga, H. (1988). Physiology in Health in Mexico: Testimonies Medical Specialties in Mexico. Compilers Soberón G, Kumate J and Laguna. Biblioteca de la Salud, Pp. 79-107.

De Michali, A. (2004). The Long Way toward Mexican Cardiology. Gaceta Medica de Mexico, 140, 340-352.

De Micheli Serra, A. (1983). Sesquicentennial of the Medical School of the National University of Mexico (Part 2: 1859-1945). Archivos de Cardiologia de Mexico, 53, 379-384.

De Micheli Serra, A., \& Izaquirre-Avila, R. (2010). European Science of the New Spain. Ciencia, 61, 22-29. 
Finch, S. (2005). Rare Books Catalogue No. 62. From the Source to the Severn Bore. Wales and England, 32/1727.

Hess, W. R. (1944). Das schlafsyndrom als folge dienzephaler reizung. Helvetica Physiologica et Pharmacologica Acta, 2 , 305-344.

Izquierdo, J. J. (1934). Quadricentennial Balance of the Mexican Physiology. México: Ediciones Ciencia, 358.

Izquierdo, J. J. (1965). William Harvey in the Motion of the Heart and Blood in Animals. México: UNAM.

Jouvet, M. (1988) The Regulation of Paradoxical Sleep by the Hypothalamo-Hypophysis. Archives Italiennes de Biologie, 126, 259-274.

Pieron, H. (1913). Le probleme physiologique du sommeil. Paris: Masson, 1-520.

Salgado, M. J. (1727). Cursus Medicus Mexicanus. México: Impr. Her. Vda de Miguel de Rivera. 